\title{
The relationship between clinical outcomes and medication adherence in difficult-to-control asthma
}

\author{
Anna C Murphy, ${ }^{1,2}$ Amandine Proeschal, ${ }^{3}$ Christopher E Brightling, ${ }^{1,2,4}$ \\ Andrew J Wardlaw, ${ }^{1,2,4}$ lan Pavord, ${ }^{1,2,4}$ Peter Bradding, ${ }^{1,2,4}$ Ruth H Green ${ }^{1,2,4}$
}

\author{
${ }^{1}$ Institute for Lung Health, \\ Leicester, UK \\ ${ }^{2}$ University Hospitals of \\ Leicester NHS Trust, Leicester, \\ UK \\ ${ }^{3}$ School of Pharmacy, Grenoble, \\ France \\ ${ }^{4}$ Department of Infection, \\ Immunity and Inflammation, \\ University of Leicester, \\ Leicester, UK
}

\section{Correspondence to}

Anna C Murphy, Consultant Respiratory Pharmacist, Department of Respiratory Medicine, Glenfield Hospital, Leicester LE3 90P, UK; anna.murphy@uhl-tr.nhs.uk

$\mathrm{PB}$ and RHG authors are Co-senior authors.

Received 12 September 2011 Accepted 29 February 2012 Published Online First 21 March 2012

\begin{abstract}
Medication non-adherence and the clinical implications in difficult-to-control asthma were audited. Prescription issue data from 115 patients identified sub-optimal adherence $(<80 \%)$ in $65 \%$ of patients on inhaled corticosteroids (ICS) or combined ICS/long-acting $\beta 2$ agonist (LABA). In those using separate ICS and LABA, adherence to LABA (50\%) was significantly better than to ICS (14.3\%). Patients with sub-optimal ICS adherence had reduced $\mathrm{FEV}_{1}$ and higher sputum eosinophil counts. Adherence ratio was an independent predictor of previous ventilation for acute severe asthma ( $p=0.008$ ) The majority of patients with difficult-to-control asthma are non-adherent with their asthma medication. Non-adherence is correlated with poor clinical outcomes.
\end{abstract}

\section{INTRODUCTION}

Asthma is one of the most common chronic conditions worldwide. Most patients with asthma have controllable disease. However, between 5\% and 10\% of patients with asthma have disease which is more difficult to control, experiencing persistent symptoms and frequent exacerbations. Morbidity and health costs are disproportionately high in these patients and they are at greater risk of fatal and near fatal exacerbations. The causes of difficult-to-control asthma are complex. In many people suboptimal adherence to prescribed medication is likely to be an important factor. ${ }^{1}$ Patient adherence to treatment is difficult to quantify. Several tools can be used to monitor adherence, including patient self-reporting, biochemical monitoring, prescription refill or issue checks, and clinician judgement. Monitoring of prescription issue data can provide clinicians with objective information that can be used to assess whether a patient is adhering to their medication regimen. This is routinely undertaken in the Difficult Asthma Clinic at Glenfield Hospital Leicester to help guide future treatment options. We have performed an audit to assess whether this mechanism of monitoring adherence in a difficult-to-control asthma population provides useful information in identifying patients with worse clinical outcomes.

\section{METHODS \\ Data}

A total of 161 adult patients with asthma attending the Difficult Asthma Clinic during July/August 2009 were included in the audit. None of the subjects were suspected of non-adherence and were selected solely on the date of their appointment at the clinic. Each patient's general practitioner (GP) was contacted to collect prescription issue data for asthma medicines over the previous 12 months. Further data were collected from the hospital's dispensing computer system over the same period. The data were collated and compared with the patient's prescribed medication regime. The number of doses per inhaler and the daily doses prescribed were taken into consideration, in addition to any changes to the patient's prescribed medication over this 12-month period. An adherence ratio was calculated as the number of doses issued divided by the number of expected issues, expressed as a percentage. Adherence to medication was defined as $\geq 80 \%$ of prescriptions issued. Demographical information collected included age, sex, smoking history, number of rescue courses of oral corticosteroid (OCS) and hospital admissions in the previous 12 months, documented previous requirement for mechanical ventilation for acute severe asthma, lung function, the modified Juniper Asthma Control Score (JACS), Hospital Anxiety and Depression scores, Nijmegen score and the induced sputum eosinophil count.

\section{Statistical analysis}

Statistical analysis was performed using SPSS for Windows. Differences between groups were compared using the independent $t$ test for continuous variables and $\chi^{2}$ analysis for categorical variables. To determine whether adherence was associated with previous ventilation for severe asthma, we performed a multivariate logistic regression analysis with previous episodes of ventilation for severe asthma as the dependent variable and the adherence ratio to inhaled corticosteroids (ICS) as a continuous independent variable. Demographical data and traditional markers of asthma severity were included in the model as covariates. The Wald $\chi^{2}$ statistic was used to determine the corrected $\mathrm{OR}$ for risk of previous ventilation associated with each $10 \%$ reduction in adherence.

\section{RESULTS}

Of the 161 patients recruited, 132 responses were received from the patients' GPs (82\%). Seventeen patients were excluded due to incomplete data recorded and a total of 115 patients were included in the audit.

\section{Adherence to asthma medicines}

Suboptimal adherence (defined as $<80 \%$ prescriptions issued) to ICS was identified in 75 of 115 
patients (65.2\%): 63 of 101 patients (62.4\%) taking combined ICS and long-acting $\beta_{2}$ agonist (LABA) inhalers and 12 of 14 patients $(85.7 \%)$ taking separate ICS and LABA inhalers $(p=0.09)$. In the 14 patients using separate ICS and LABA inhalers, adherence to the LABA (50\%) was significantly better than adherence to the ICS $(14.3 \%)(p=0.043)$. Similar proportions of patients were suboptimally adherent to leukotriene antagonists (30 of 42, 71.4\%), antimuscarinic inhalers (22 of 29, $75.9 \%$ ) and oral theophylline (23 of $40,57.5 \%$ ). Fewer patients were found to have suboptimal adherence to oral corticosteroid (OCS) (13 of 50, 26\%) ( $<<0.0001)$. Only 29 of $115(25.2 \%)$ patients were found to be adherent to all prescribed medication. No significant differences in age, gender, racial origin, smoking history or prescribed doses of ICS or OCS were seen. There was no significant association between adherence with ICS and OCS $(p=0.48)$, suggesting that the suboptimal adherence with ICS was not explained by good adherence with OCS.

Ninety-eight patients were prescribed both ICS and shortacting $\beta_{2}$ agonists (SABAs). Forty-eight of these patients $(41.7 \%)$ filled more prescriptions for their SABA than for ICS.

\section{Asthma control}

Patients with suboptimal adherence to ICS had a lower postbronchodilator forced expiratory volume in $1 \mathrm{~s}\left(\mathrm{FEV}_{1}\right)(\mathrm{p}=0.049)$, and higher $\%$ sputum eosinophil counts $(p=0.05)$ than those who had adequate ICS adherence. There were no significant differences in symptom scores, hospital anxiety and depression scores, or Nijmegen scores between the two groups (table 1).

\section{Healthcare utilisation}

There were no significant differences in the number of rescue courses of OCS in the previous year or the rate of previous admissions to hospital or to an intensive treatment unit for asthma between the two groups, but patients with suboptimal ICS adherence were more likely to have been ventilated for asthma $(p=0.02)$. In a multivariate logistic regression model with adherence ratio as a continuous variable, including demographical data (age, gender, pack years smoked) and measures of asthma control (ICS dose, maintenance OCS use, \% predicted post-bronchodilator $\mathrm{FEV}_{1}$ and modified JACS score), the adherence ratio was the only independent predictor of previous need for ventilation for acute severe asthma (OR 0.054 (95\% CI 0.01 to 0.47$), p=0.008)$. This means that for each $10 \%$ decrease in adherence to ICS, the estimated odds of having been ventilated for asthma increased by 1.35 times.

\section{DISCUSSION}

We confirmed the findings of a recent study that adherence to ICS in a population with difficult-to-control asthma is suboptimal and is accompanied by significant adverse outcomes. ${ }^{1}{ }^{2}$ In addition, our audit demonstrates for the first time that suboptimal adherence to asthma medications is associated with a history of mechanical ventilation. The logistic regression analysis result demonstrates that the adherence ratio is an independent predictor of previous need for mechanical ventilation for acute asthma. Since the need for mechanical ventilation represents near death, it is interesting that this finding is similar to the epidemiological data of Suissa et al, which showed that the rate of death from asthma among users of ICS decreased by $21 \%$ for every additional canister of ICS used during the year. ${ }^{3}$ This, coupled with our logistic regression analysis, suggests that it is most likely that suboptimal adherence increases the risk of ventilation rather than patients stopping treatment after being ventilated because they thought the medication did not work. Furthermore, we found that the use of combined inhalers containing ICS and LABA appears to improve adherence to ICS compared with the use of individual inhalers. There is current concern about the use of LABAs, particularly if they are used in the absence of an $\mathrm{ICS}^{4}$ Current guidelines recommend that LABAs are only used in conjunction with an ICS and to prevent the use of LABA alone it has been suggested that patients should only be prescribed combined inhalers containing both ICS and LABA. Our data provide evidence to support this recommendation. Although the numbers were small, there was a clear trend towards lower adherence to ICS in patients using separate inhalers compared with combined inhalers, and worryingly, those patients using separate ICS and LABA inhalers were significantly more adherent to the LABA than the ICS.

Suboptimal adherence to prescribed medication regimens is a common feature of chronic disease. ${ }^{5}$ It is an important issue as there is the potential for patients to suffer adverse diseaserelated outcomes through the failure to take medication, or adverse drug effects due to escalation of treatment due to apparently failing drug regimens. How to assess adherence and whether it is important in terms of clinical outcomes is not well defined. The simplest method to identify non-adherence is to ask the patient, but many patients are reluctant to inform their physicians that they did not follow medical instructions. Patients may also overestimate adherence through forgetfulness or because they have a memory bias towards days when they were adherent. Measuring of adherence by prescription issue rates provides an objective measure, and is a simple and practical tool to use in the clinical setting. ${ }^{1}$ The information is readily available from the GP and hospital pharmacy allowing a picture of the patient's adherence to be quickly presented. It is robust in that a failure to collect prescriptions means the patient will not have taken their medication, but it may overestimate adherence as patients may still collect their medication but not take it. There is no consensual standard for what constitutes adequate adherence. Few studies have examined the relationship between adherence and outcomes to answer this. We feel that $80 \%$ is

Table 1 Patient clinical outcomes according to their prescription adherence ratio to inhaled corticosteroid (ICS) (adherence ratio $<0.8$ vs $\geq 0.8$ )

\begin{tabular}{|c|c|c|c|}
\hline All patients $(n=115)$ & $\begin{array}{l}\text { ICS adherence ratio } \\
<0.8(\mathrm{n}=75)(\text { mean }(\mathrm{SD}))\end{array}$ & $\begin{array}{l}\text { ICS adherence ratio } \\
\geq 0.8(n=40)(\text { mean }(S D))\end{array}$ & p Value \\
\hline Post bronchodilator $\mathrm{FEV}_{1} \%$ predicted & $75.5(20.9)$ & $84.3(23.5)$ & 0.049 \\
\hline Sputum eosinophil count (\%) & $4.6(0.7)$ & $2.3(0.5)$ & 0.05 \\
\hline Modified JACS & $2.25(1.2)$ & $2.05(1.4)$ & 0.50 \\
\hline Hospital anxiety score & $7.5(4.4)$ & $7.1(4.6)$ & 0.69 \\
\hline Hospital depression score & $6.1(4.2)$ & $5.2(3.8)$ & 0.32 \\
\hline Nijmegen score & $15.3(14.1)$ & $18.8(14.9)$ & 0.22 \\
\hline
\end{tabular}

$\mathrm{FEV}_{1}$, forced expiratory volume in $1 \mathrm{~s}$; JACS, Juniper Asthma Control Score. 
a sensible cutoff because it allows for realistic expectations of patient behaviour while ensuring clinical efficacy of the drug.

In summary, this audit shows that in this difficult-tocontrol asthma population suboptimal adherence is common, and associated with adverse clinical outcomes and an increased risk of near-fatal asthma. Measuring adherence objectively using prescription issue data is therefore worthwhile and readily achieved in routine clinical practice. In our view, it is essential in the assessment of patients with difficult-to-control asthma, particularly when costly or potentially toxic therapies are being considered. The challenge now is to develop patient-orientated strategies that improve medication adherence, which in turn should improve the clinical outcome in this group of patients with difficult-tocontrol asthma.
Acknowledgements We would like to thank the multidisciplinary team working in the Difficult Asthma Clinic at Glenfield Hospital, Leicester, for their support with this project. We would also like to thank Mary Linnett, medical secretary at UHL.

Competing interests None.

Provenance and peer review Not commissioned; externally peer reviewed.

\section{REFERENCES}

1. Gamble J, Stevenson M, McClean E, et al. The prevalence of non-adherence in difficult asthma. Am J Respir Crit Care Med 2009:180:817-22.

2. Suissa S, Ernst $P$, Kezouh A. Regular use of inhaled corticosteroids and the long term prevention of hospitalisation for asthma. Thorax 2002;57:880-4.

3. Suissa S, Ernst P, Benayoun S, et al. Low-dose inhaled corticosteroids and the prevention of death from asthma. N Engl J Med 2000;343:332-6.

4. Hasford J, Virchow JC. Excess mortality in patients with asthma on long-acting beta2-agonists. Eur Respir J 2006;28:900-2.

5. Horne R. Compliance, adherence and concordance; implications for asthma treatment. Chest 2006;130(1 Suppl):65S-72S.

\section{Have confidence in your decision making.}
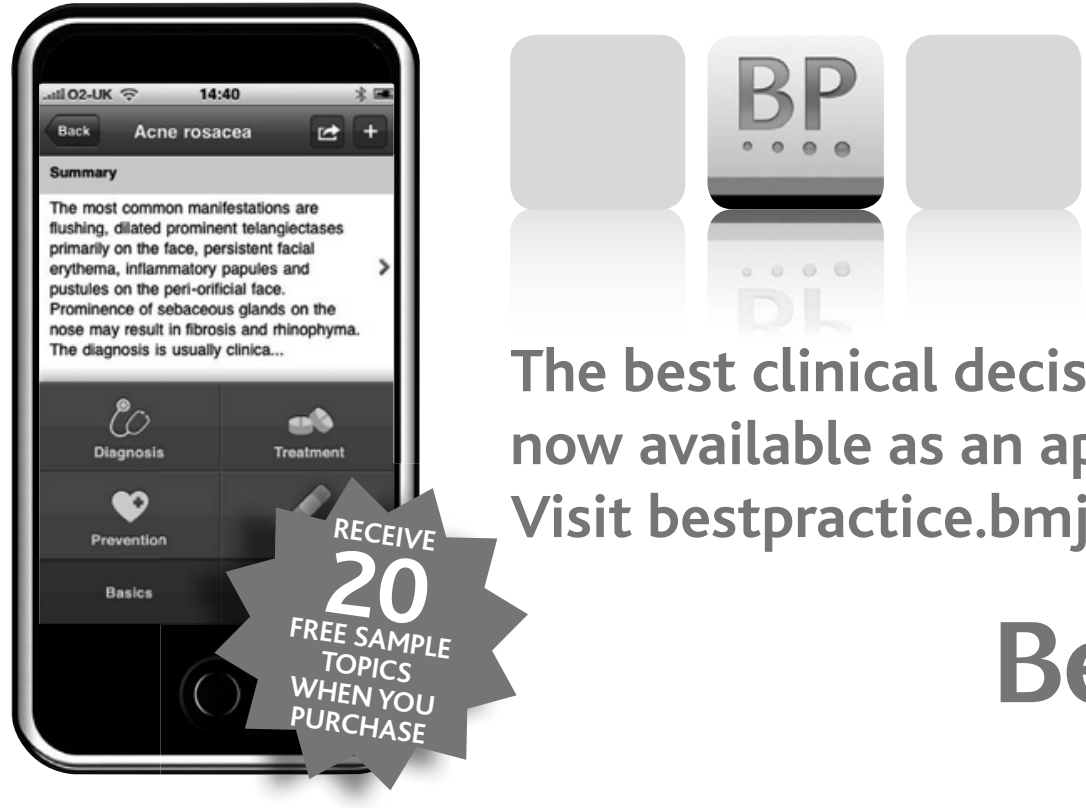

The best clinical decision support tool is now available as an app for your iPhone. Visit bestpractice.bmj.com/app

\section{clinicians $\bullet$ medical students $\bullet$ nurses $\bullet$ healthcare practitioners}

